\title{
Focal Sweep Videography with Deformable Optics
}

\author{
Daniel Miau \\ Columbia University \\ dmiau@cs. columbia.edu
}

\author{
Oliver Cossairt \\ Northwestern University \\ olliedeecs.northwestern.edu
}

\author{
Shree K. Nayar \\ Columbia University \\ nayarecs.columbia.edu
}

\begin{abstract}
A number of cameras have been introduced that sweep the focal plane using mechanical motion. However, mechanical motion makes video capture impractical and is unsuitable for long focal length cameras. In this paper, we present a focal sweep telephoto camera that uses a variable focus lens to sweep the focal plane. Our camera requires no mechanical motion and is capable of sweeping the focal plane periodically at high speeds. We use our prototype camera to capture EDOF videos at $20 \mathrm{fps}$, and demonstrate space-time refocusing for scenes with a wide depth range. In addition, we capture periodic focal stacks, and show how they can be used for several interesting applications such as video refocusing and trajectory estimation of moving objects.
\end{abstract}

\section{Introduction}

The depth of field (DOF) of an imaging system is the range of depths over which scene points appear sharp in an image. In many applications, such as microscopy and surveillance, it is often desirable to have a very large DOF. The lens of any imaging system, however, limits its DOF. One can increase the DOF by reducing the size of the aperture, however, at the expense of reducing the signal-to-noise ratio (SNR) of captured images. Focal Sweep has been proposed as a technique to extend the DOF of an imaging system while maintaining high SNR $[8,11,15,24,22]$.

Focal sweep using mechanical motion was originally proposed by Hausler [8] to extend the DOF of microscopes (i.e. scenes with depth ranges on the order of 10's to 100's of $\mu \mathrm{m}$ ). More recently, the idea has been extended for larger depth ranges that are encountered in conventional photography (e.g. scenes with depth ranges on the order of 10's to 100's of $\mathrm{cm}$ ) [11]. Telephoto imaging (e.g., scenes with depth range on the order of 10's to 100's of meters) is an area where focal sweep could be particularly useful because telephoto lenses typically have a narrow DOF. Large sensor travel, however, poses serious practical issues. More fundamentally, mechanical motion makes video capture impractical. We discuss these issues in Section 3. In this work, we

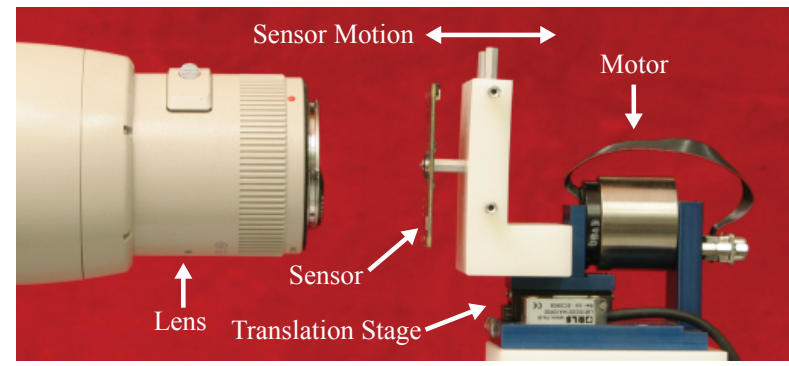

(a) A telephoto focal sweep camera with a moving sensor

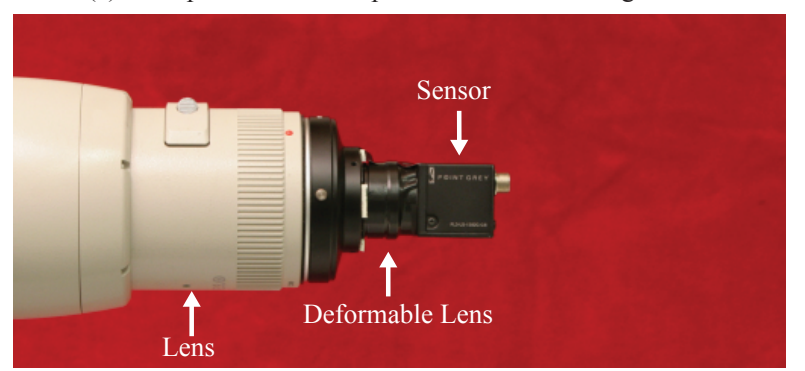

(b) A telephoto focal sweep camera with a deformable lens

Figure 1. Focal sweep with a moving sensor versus a deformable lens. (a) and (b) show two implementations of a telephoto focal sweep system using a $800 \mathrm{~mm}$ lens. (a) shows an implementation with a moving sensor. Note the complexity and the size of the mechanism required to translate the sensor at high speed. The long sensor travel poses significant engineering challenges, such as susceptibility to vibration. (b) shows a compact implementation with a tunable lens. In this paper, we show several interesting applications enabled by focal sweep with deformable optics.

seek to overcome these limitations by integrating a variable focal length lens into an imaging system to demonstrate focal sweep videography.

For a conventional singlet lens, focal length is fixed at manufacture time. In contrast, the focal length of a variable focal length lens can be controlled dynamically. There are two common approaches to implement variable focal length lenses: the deformable surface approach and the variable index of reflection approach. In the deformable surface approach, the lens shape is deformed based on the electrowetting effect [23] or a liquid/membrane principle [19]. 
In the variable index of reflection approach, lens shape is fixed and the index of refraction is controlled by applying an electric field [21]. We focus our attention on the lenses based on the deformable surface approach (which we refer to as deformable lenses) due to their larger aperture size and superior dynamic response. While deformable lenses have just begun to make their way into mass-produced consumer imaging systems, they have long played a crucial role in biological vision. For instance, human eyes do not focus by moving the position of the lens, but by using the ciliary muscle to adjust lens curvature [12,9].

The following are the main contributions of this paper.

Telephoto focal sweep camera. We present a telephoto focal sweep camera built from off-the-shelf parts. We use a deformable lens to sweep the focal plane (see Figures 1(b) and 4(b)). A comparable swept-sensor system requires a high power voice coil actuator and translation stage (see Figure 1(a)) which introduces significant vibrations when operating at high frequencies.

Extended DOF video. Previous focal sweep research was mostly restricted to static scenes. In this project, we demonstrate a telephoto focal sweep camera which is capable of capturing EDOF videos at $20 \mathrm{fps}$.

Periodic focal stack. For EDOF videos, we use periodic focal plane motion and capture an image every half-period. By simply increasing the frame rate of the camera, we can capture a periodic focal stack. From a periodic focal stack we can generate refocusable videos (see Figures 2) and estimate the 3D trajectory of moving objects. We believe this system will be useful for surveillance, security, and entertainment applications.

Space-time refocusing. We demonstrate the use of the telephoto focal sweep camera for space-time refocusing [26] and show several refocusing examples.

\section{Related Work}

Single-Shot Extended Depth of Field (EDOF). DOF can be extended by placing an optical element in the lens aperture to engineer the camera point spread function (PSF). An EDOF image is then recovered by deconvolving the captured image with either a depth-invariant $[6,5,4]$ or depth-dependent $[13,14,25]$ blur kernel. The major advantage of the above techniques is that no moving parts are required. The disadvantage is that DOF is fixed at the time of configuring the imaging system and is cumbersome to change. While the above techniques are suitable for shortfocal length cameras, they are costly to manufacture and error-prone for large focal length systems due to the large aperture sizes involved.

Focal sweep cameras $[8,11]$ also produce depthindependent blur that can be removed via deconvolution. Recent works by Liu et al. [15] and Zhao et al. [24] use a deformable lens to extend DOF. However, the $15 \mathrm{fps}$ EDOF video demonstrated in [15] is from a microscopic static scene (cactus thorns), in addition, the cameras in both papers can only be used to image close scenes $(<100 \mathrm{~cm}$ depth) with relatively small depth ranges $(<10 \mathrm{~cm})$. In contrast, our telephoto focal sweep camera can extend DOF for distant scenes $(>50 \mathrm{~m})$ with much larger depth ranges $(>10 \mathrm{~m})$. In addition, we demonstrate how to capture EDOF videos of dynamic scenes, refocusable photographs and periodic focal stacks.

Focal Stacks and Refocusing. A focal stack is a sequence of images captured with different focus settings. Several researchers have shown how a light field can be used to generate refocusable photographs (a focal stack) [10, 17]. Light field capture enables digital refocusing from a single snapshot, however this comes at the cost of a sacrifice in spatial resolution.

By sweeping the focal plane, a focal stack can be captured without sacrificing spatial resolution. Frames within the stack, however, must be captured sequentially. A focal stack can be used to extend DOF: an all-in-focus image can be synthesized by extracting the focused region within each image of the focal stack and compositing into a single image $[1,7]$. Shroff et al. used periodic focal stacks to recover EDOF videos at $30 \mathrm{fps}$, but their technique relies on depth estimation and sensor motion, which is impractical for telephoto systems. Focal stack capture can cause problems for dynamic scenes with fast moving objects, however this can be exploited for the purpose of space-time refocusing, introduced by Zhou et al. [26].

\section{Practical Issues with Swept-Sensor Focal Sweep}

A motion-based focal sweep camera sweeps the focal plane by translating either the sensor, lens, or object along the optical axis during image capture $[8,11]$. Without loss of generality, we assume sensor motion. We now show that the required travel distance of the sensor increases significantly with focal length.

Given a lens of focal length $f$ and a scene point $s$ at distance $o$, the Gaussian thin lens law states that a focused image of $s$ can be formed at the distance $i$ :

$$
i=1 /\left(\frac{1}{f}-\frac{1}{o}\right),
$$

Let $o_{1}$ and $o_{2}$ be the two extents of the depth range and $i_{1}$ and $i_{2}$ be their corresponding image distances. To sweep the focal plane from $o_{1}$ to $o_{2}$, the sensor needs to be translated from $i_{1}$ to $i_{2}$. It can be shown using the thin lens law that the travel distance $d$ of the sensor is:

$$
d=\left|i_{2}-i_{1}\right|=\left|\frac{f^{2}\left(o_{1}-o_{2}\right)}{\left(o_{1}-f\right)\left(o_{2}-f\right)}\right|,
$$


(a)

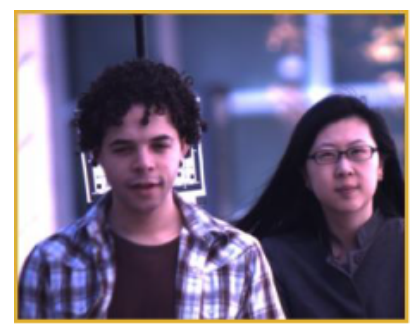

(d)

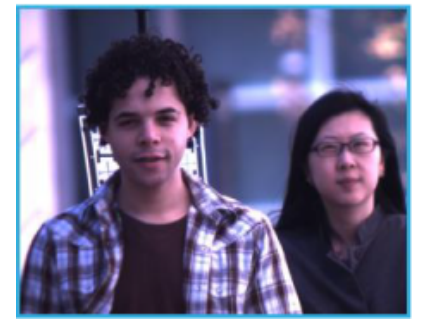

(b)

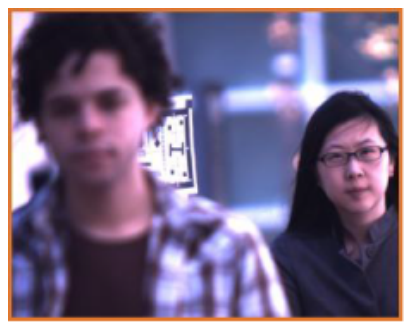

(e)

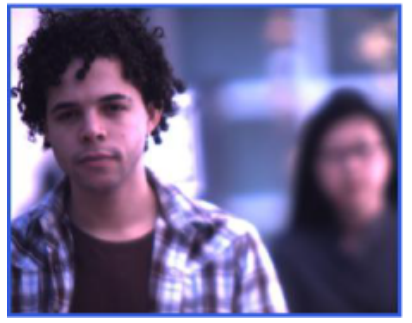

(c)

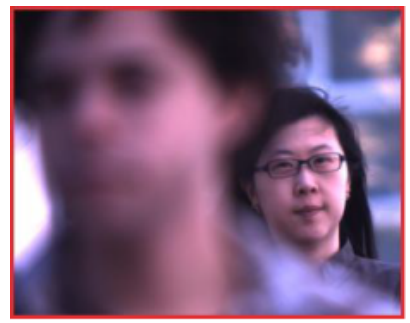

(f)

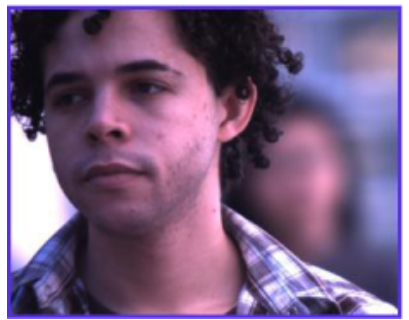

(g)

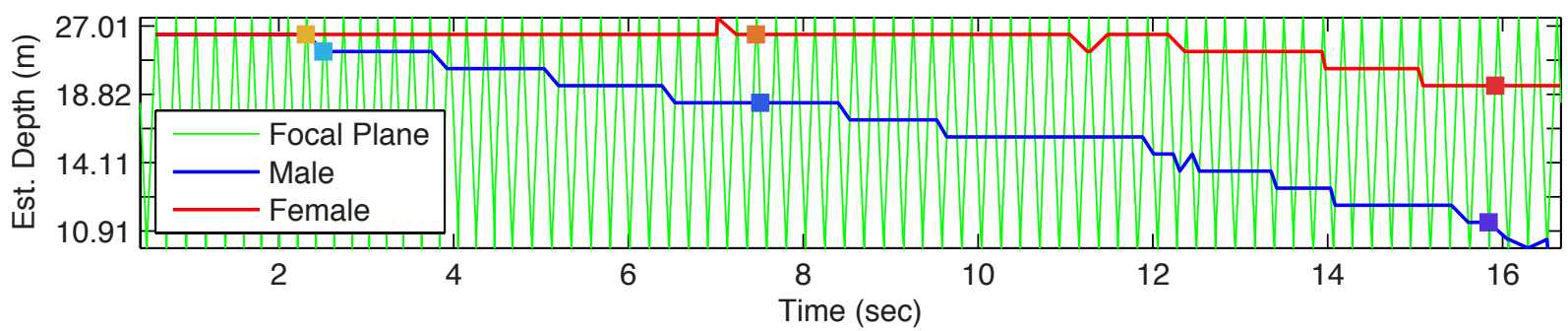

Figure 2. Two refocused videos generated from a periodic focal stack. In this example the periodic focal stack includes two people walking toward the camera at different speeds. $(a, b, c)$ show three frames of a generated video in which the person on the right is kept in focus. (d, e, f) show three frames of a generated video in which the person on the left is kept in focus. (g) shows the estimated depths of the focal plane (green line) and the two people (blue and red lines) over time. By selecting the frames captured at the times when the focal plane coincides with the depth of a person, one can generate a video in which focus follows the person. The colored markers indicate the locations corresponding to the frames shown above. Periodic focal stacks are discussed in Section 5.2.

When $o_{1}, o_{2} \gg f, d$ is proportional to $f^{2}$. For example, to cover the depth range of $[60 \mathrm{~m}, 70 \mathrm{~m}]$, a $12.5 \mathrm{~mm}$ focal sweep system [11] needs to translate its sensor by $37.2 \mu \mathrm{m}$. In comparison, the travel distance of a $800 \mathrm{~mm}$ and a $2000 \mathrm{~mm}$ focal sweep systems would be $1562.3 \mu \mathrm{m}$ and $10142 \mu \mathrm{m}$, respectively. This corresponds to an increase of the travel distance by factors of 4198 and 22763.

Thus far the focal sweep imaging systems reported in the literature typically use small focal length lenses. It can be a challenge to translate a sensor by even the moderate distances reported in $[11,22]$ at frequencies greater than a few cycles per second. Kuthirummal et al. demonstrated an EDOF video at $1.5 \mathrm{fps}$ captured with a $12.5 \mathrm{~mm}$ focal length lens. They stated that the frame-rate is limited by the actuator performance. This problem increases quadratically with focal length. A voice coil actuator can be used to achieve large amplitude periodic motion (see Figure 1(a)). However, large amplitude, high frequency motion will induce sensor vibration. If these vibrations cannot be isolated from the sensor and lens, image quality will suffer. Indeed, we tried using the voice coil system in Figure 1(a) to capture EDOF videos, but the captured frames had significant motion blur. In the following section we show that a deformable lens is particularly well suited for implementing focal sweep at video rates even with a large focal length lens.

\section{Focal Sweep System with Deformable Optics}

We first show that a camera with a single deformable lens can be used for focal sweep. Following this analysis, we describe our prototype camera which we model as a compound system with two thin lenses. We explain the design decisions which led us to build this particular prototype. We conclude this section by describing how to achieve the optimal integrated PSF (IPSF), and use simulations and experiment results to verify our analysis.

\subsection{Deformable Lens Focal Sweep Analysis}

Figure 3 illustrates a simple focal sweep camera with a single deformable lens. The sensor is fixed at a distance $i$ from the lens. Consider a scene point $s$ at distance $o$ from the lens. A focused image of $s$ is produced at location $m$ on the sensor when the focal length of the deformable lens is $f$. When the focal length of the deformable lens changes to 
(a)

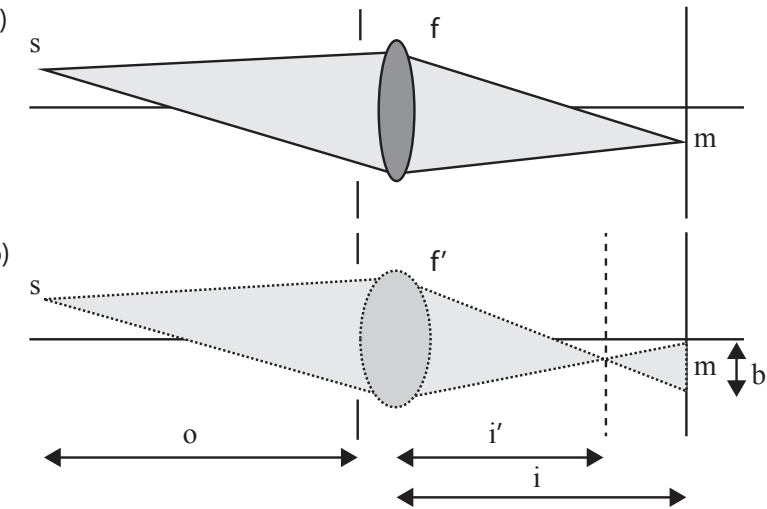

Figure 3. Focusing for a deformable lens. (a) and (b) show rays traced through the same deformable lens with different focal lengths. (a) A scene point $s$, at a distance $o$ from the lens, is imaged in perfect focus at location $m$ on a sensor at a distance $i$ from the lens. (b) If the focal length of the lens is changed such that an image in perfect focus is at a distance $i^{\prime}$ from the lens, $s$ is imaged as a blurred circle with diameter $b$ centered around $m$.

$f^{\prime}$, the image of $s$ will be a blur disk (circle of confusion) with diameter $b$ given by

$$
b=\frac{a}{i}\left(i-i^{\prime}\right),
$$

where $i^{\prime}=1 /\left(\frac{1}{f^{\prime}}-\frac{1}{o}\right)$. The sign of $b$ indicates whether a focused image is formed in front of or behind the sensor. From Equation 3 we know that $b$ can be controlled by varying the focal length. The distribution of light energy within the blur circle is referred to as the point spread function (PSF), which can be parameterized as $P(r, o, f(t))$ [11], where $r$ is the radial position on the sensor plane. When the focal length of the deformable optics is varied during exposure, the IPSF is determined as

$$
I P(r, o)=\int_{0}^{T} P(r, o, f(t)) d t,
$$

where $T$ is the total exposure time. For a swept-sensor system, the motion trajectory of the sensor can be programmed to achieve the optimal IPSF. The optimal motion trajectory has been shown to be constant sensor motion $[2,11]$. In a deformable focal sweep system, the time-varying focal length $f(t)$ determines the shape of IPSF. The key to achieving the optimal IPSF is to ensure that the blur diameter varies linearly as a function of time [2]. It is straightforward to show that when imaging distant objects, the optimal IPSF for a single deformable lens focal sweep system is achieved when $f(t)$ is varied linearly as a function of time.

\subsection{Prototype Camera}

Our prototype is shown in Figures 1(b) and 4(b). It consists of a Canon $800 \mathrm{~mm}, f / 5.6$ lens, an Optotune EL-1030 tunable lens, an Optotune signal generator to control the

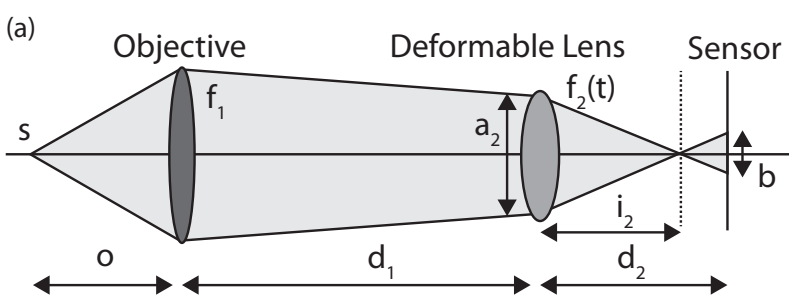

(b)

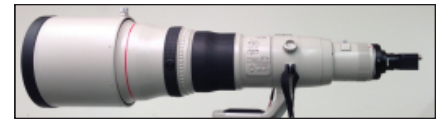

Figure 4. Prototype telephoto focal sweep camera. (a) We use two thin lenses to model the PSF of the telephoto focal sweep camera shown in (b). The parameters in the figure are discussed in Section 4.2. The prototype camera consists of a Canon $800 \mathrm{~mm}$, $f / 5.6$ lens, an Optotune EL-10-30 tunable lens, and a Point Grey Flea3 image sensor.

deformable lens, and a Point Grey Flea3 image sensor. The signal generator is capable of outputting triangular and sinusoidal drive signals. The signal amplitude can be adjusted so that the focal length varies anywhere between $40 \mathrm{~mm}$ and $120 \mathrm{~mm}$ and the drive frequency can be adjusted from $0 \mathrm{~Hz}$ to $340 \mathrm{~Hz}$. However, the amplitude of the frequency response falls off as the drive frequency increases. The tunable lens is attached to the mounting flange of the Canon lens and the sensor is mounted to the back of the deformable lens.

Compound thin lens model. Because we don't have access to the Canon lens prescription, we model our implementation as a system of two thin lenses: one objective, and one deformable lens. The thin lens model approximates our system reasonably well because the Canon lens is highly corrected for geometric aberrations. In practice, we observed that most geometric aberrations are minimal except a moderate amount of geometric distortion. Note that these aberrations could be further minimized by placing the deformable lens in the pupil plane of the telephoto lens. However, this solution only works for small focal length imaging systems $[15,24]$ since deformable lenses cannot be manufactured with large aperture sizes $(10 \mathrm{~mm}$ is the maximal aperture size at the time of this writing [3]). As a result, we are required to use a large separation between the principal plane of the telephoto lens and the deformable lens.

Optimal focal length profile. We now show how to derive the time-varying focal length that optimizes the IPSF of our system. Figure 4 shows the optical layout used to model our system. The system consists of an objective of fixed focal length $f_{1}$, a deformable lens with time-varying focal length $f_{2}(t)$ and aperture diameter $a_{2}$. The distance between the objective and the deformable lens is $d_{1}$. The distance between the sensor and the deformable lens is $d_{2}$. 


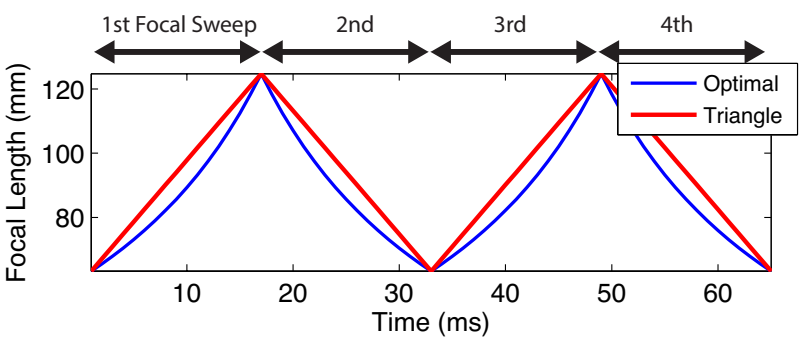

(a) Optimal and Triangle Profiles

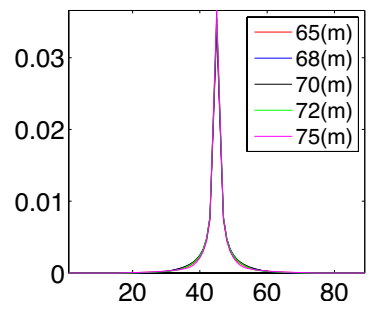

(b) Optimal IPSF

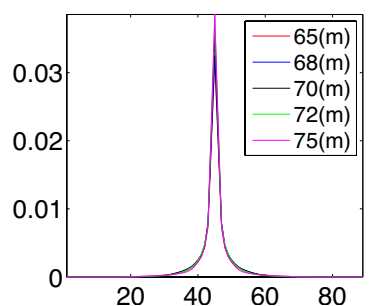

(c) Triangle IPSF
Figure 5. Focal length profiles and resulting IPSFs. (a) The estimated optimal focal length profile (blue) versus the triangular approximation (red) for the prototype camera used to capture the results shown in Figures 6 and 7. (b) The IPSF produced by the optimal profile for different depths. Note the IPSF is almost completely depth-invariant. (c) The IPSF produced by the triangular profile. Note that the triangular IPSF is almost identical to the optimal one in (b). A triangular profile was used for most of the experiments in this paper.

The diameter of the blur disk $b$ is:

$$
b=\frac{a_{2}}{d_{2}}\left(d_{2}-i_{2}(t)\right)
$$

where

$$
i_{2}(t)=\frac{f_{2}(t) d-f_{2}(t) o f_{1} /\left(o-f_{1}\right)}{d-f_{2}(t)-o f_{1} /\left(o-f_{1}\right)},
$$

is the separation between the deformable lens and the plane of focus [9]. Again, $b$ can be controlled by $f_{2}(t)$. To ensure that the blur diameter varies linearly as a function of time, we take the time derivative of Equation 5 and set it equal to a constant value $\gamma$, which in turn can be determined by the desired depth range and exposure time. The result is a non-linear differential equation in $f_{2}(t)$ with solution:

$$
f_{2}(t)=\frac{\alpha \beta t+\alpha \kappa-1}{\beta t+\kappa},
$$

where $\alpha=d_{1}-\frac{o f_{1}}{o-f_{1}}, \beta=\frac{\gamma}{\alpha^{2}}, \kappa=\frac{1}{\alpha-f_{2}(0)}$. Note that $f_{2}(t)$ is independent of depth $o$ when imaging objects at distances much larger than the objective focal length (i.e. $o \gg f_{1}$ ). The time-varying focal length $f_{2}(t)$ can be highly nonlinear, depending on the focal sweep range. However,
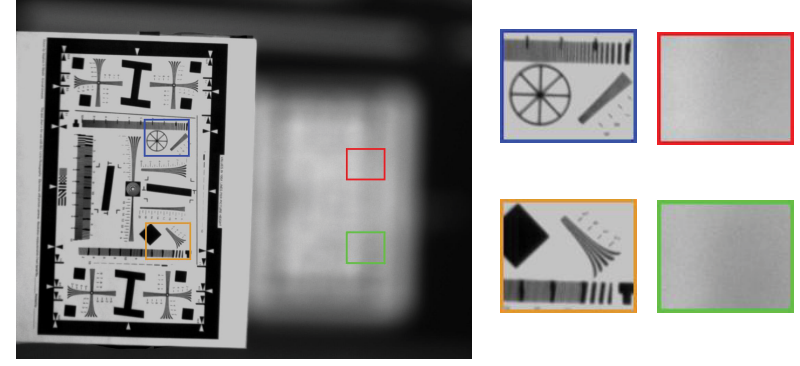

(a) Focus fixed on foreground
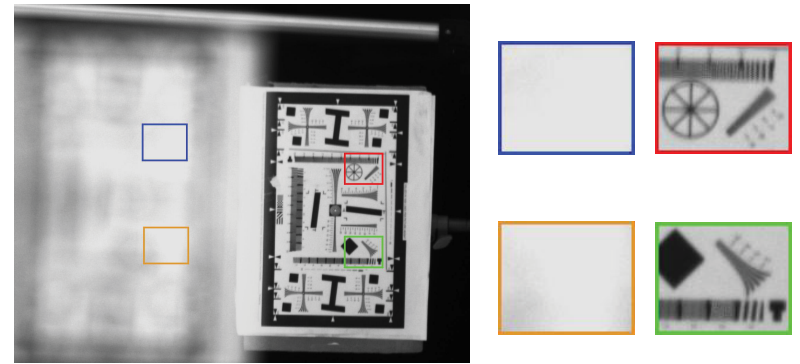

(b) Focus fixed on background
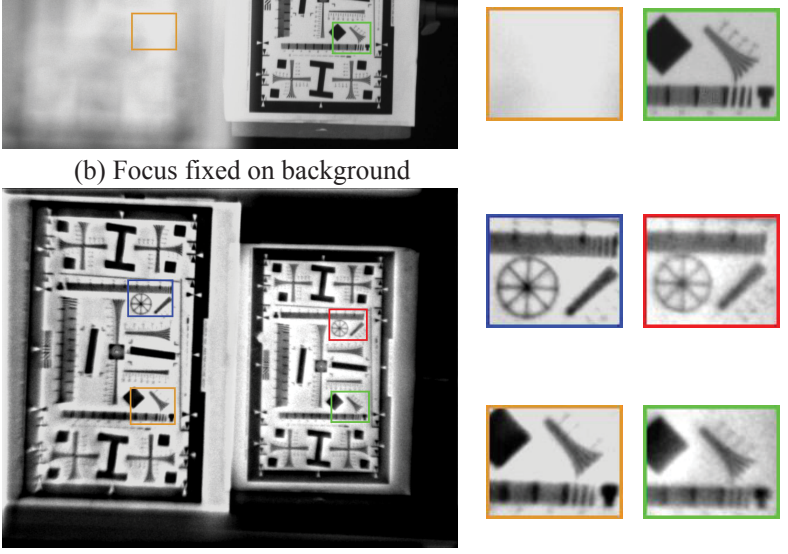

(c) Focal sweep image after deblurring
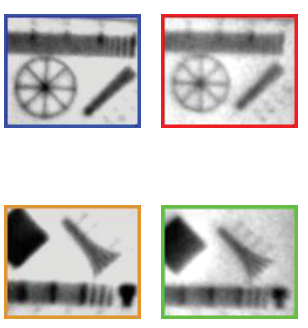

Figure 6. Comparison between two fixed focus images and a focal sweep image. The left and right resolution charts are placed at distances of $65 \mathrm{~m}$ and $75 \mathrm{~m}$ away from the camera, respectively. (a) An image captured with focus fixed on the left resolution chart. (b) An image captured with focus fixed on the right resolution chart. (c) A focal sweep image after deblurring. For the fixed focus images, the DOF is so narrow that one of the two charts is blurred beyond recognition. The insets at the lower right show that details from both resolution charts are preserved in the EDOF image

for a smaller focal sweep range, the curve can be well approximated by a straight line. Figure 5 shows the optimal time varying focal length to drive the deformable lens for $f_{1}=800 \mathrm{~mm}, d_{1}=790.68 \mathrm{~mm}$, and $d_{2}=15.26 \mathrm{~mm}$, corresponding to the estimated parameters of the prototype camera used to capture the results shown in Figures 6 and 7. Note that the optimal curve closely approximates a triangular signal. Figure 5 shows that the optimal IPSF and triangular IPSF are nearly identical.

Verification. To verify that our system can be used to extend depth of field, we captured images of a scene consisting of two resolution charts placed at a distance of $65 \mathrm{~m}$ and $75 \mathrm{~m}$. We used the focal sweep system from Figure 4(b) to capture images. Figure 6 shows the results. The top two 

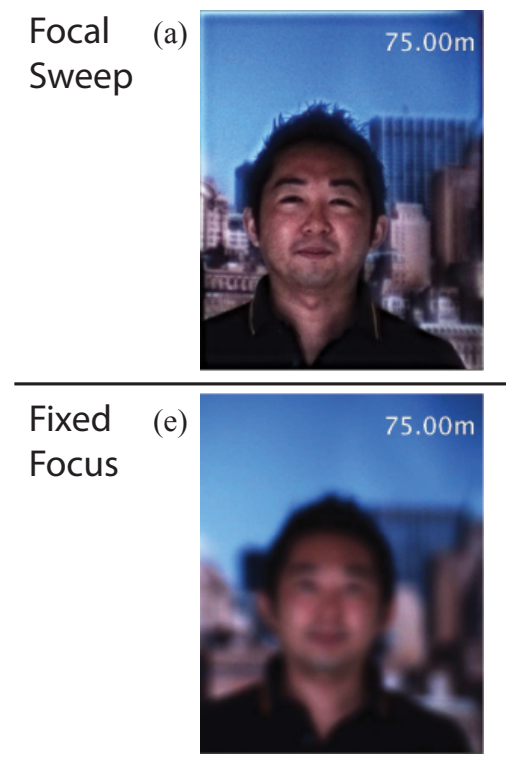

(b)

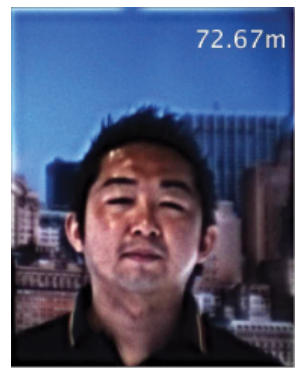

(f)

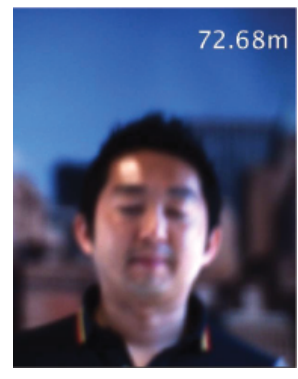

(c)

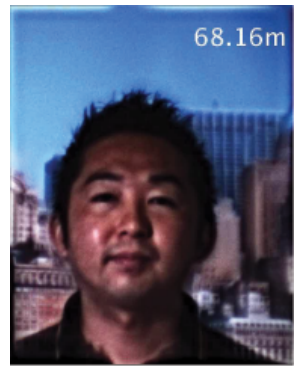

(g)

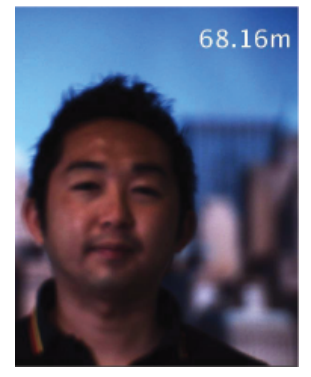

(d)

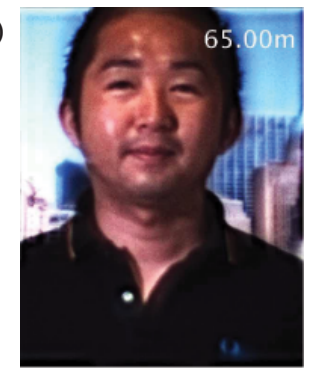

(h)

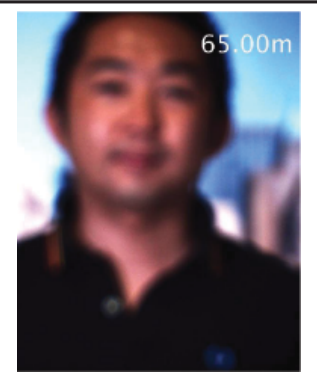

Figure 7. Comparison between a fixed focus video and a focal sweep video. The person in the scene walked from $75 \mathrm{~m}$ to $65 \mathrm{~m}$ away from the camera. ( $a, b, c, d$ ) show four frames of the focal sweep video (after deblurring). (e, f, g, h) show four frames of the video with focus fixed at $70 \mathrm{~m}$. The numbers in the upper right corner of the frames indicate the distances of the person.

images show the images captured with a fixed focus. The DOF is so narrow that the details from only one resolution chart are visible at a time. The bottom figure shows the results when sweeping focus from $65 \mathrm{~m}$ to $75 \mathrm{~m}$ during exposure. The captured image was deblurred using Weiner deconvolution and the IPSF was estimated using the system parameters. The insets on the lower right show that details from both resolution charts are indeed preserved in the EDOF image.

\section{Applications}

\subsection{Extended Depth of Field Video}

We used the prototype focal sweep camera to capture two videos of a person walking from $75 \mathrm{~m}$ to $65 \mathrm{~m}$ away from the camera. For the first video, the focus was swept periodically from $65 \mathrm{~m}$ to $75 \mathrm{~m}$. For the second video, the focus was fixed at a distance of $70 \mathrm{~m}$ away from the camera. Both videos were captured at $20 \mathrm{fps}$ with $50 \mathrm{~ms}$ exposure time for each frame.

In principle, it is not necessary to synchronize the sensor and deformable lens as long as the deformable lens is actuated at frequencies that are a multiple of half the framerate. This will ensure that each video frame will integrate over an entire focal sweep range. In practice we found that performance was good for any sweep frequency resulting in multiple focus sweeps per exposure. We found that $30 \mathrm{~Hz}$ gave the best performance. We estimated the IPSF using the system parameters and deblurred captured video frames using Weiner deconvolution.
Figure 7 shows the comparison between a video captured with a fixed focus setting and a focal sweep video. The top and bottom rows show performance with and without focal sweep, respectively. Without focal sweep the face is blurred significantly at distances away from the focal plane. With focal sweep, the face is well-focused over the entire depth range. Video results described in this section can be seen at [20]. Our results exhibit some image artifacts, possibly caused by lens aberrations, synchronization issues, and PSF estimation error.

\subsection{Periodic Focal Stacks}

A periodic focal stack is a sequence of images captured while a camera sweeps the focal plane periodically. When the frequency is high enough, each focal stack is captured almost instantaneously. As a result, a periodic focal stack is a richer representation of a scene than a traditional video.

We captured a scene of two people walking at different speeds towards the camera over a depth range from $10 \mathrm{~m}$ to $27 \mathrm{~m}$, with a system similar to the one described in Section 4.2 (the $800 \mathrm{~mm}$ lens is replaced by a $200 \mathrm{~mm}, \mathrm{~F} / 2.8$ lens for a wider field of view). To have a sufficient number of frames $(\approx 14)$ per focal stack, the focal plane sweeping frequency $(\approx 4.3 \mathrm{~Hz})$ was limited by the maximal camera frame rate $(120 \mathrm{fps})$. In the following we describe two periodic focal sweep applications from our experiment: depth tracking of moving people and refocusable videos.

Depth tracking of moving people. Depth tracking consists of three steps. We first use the focus measure of a sta- 


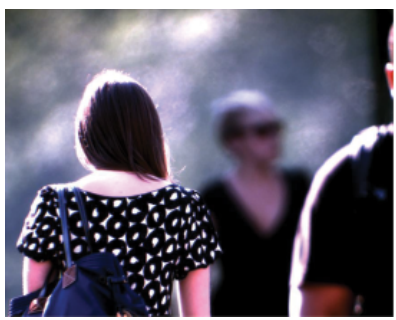

(a) Focus on foreground

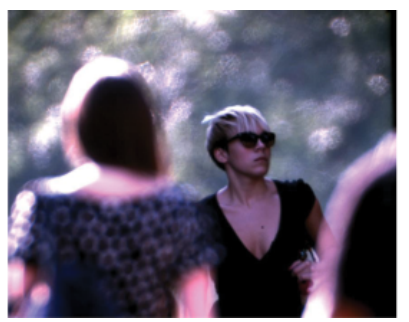

(b) Focus on middle

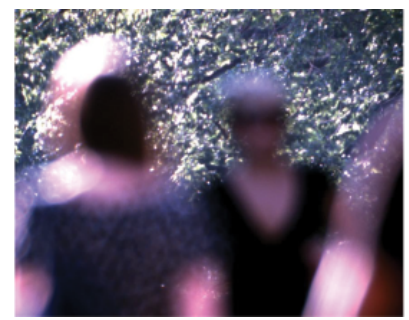

(c) Focus on background

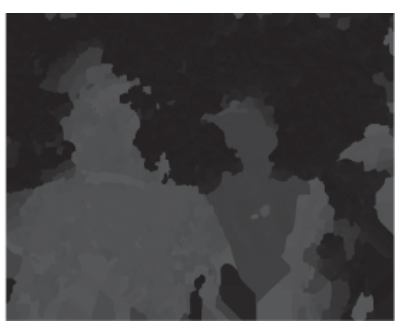

(d) Index Map

Figure 8. An example of space-time refocusing. ( $a, b, c)$ show three different refocusing results. The scene distance is roughly from $200 \mathrm{~m}$ to $280 \mathrm{~m}$. The DOF is very narrow, but everything in the scene comes into focus in at least one frame. As the scene is refocused, objects move from frame to frame. (d) shows the index-map [26] used to facilitate refocusing.

tionary object to partition the captured periodic focal stack into a sequence of focal stacks. This is necessary because our system lacks synchronization between camera and deformable lens.

Next, we run the Omron face detector [18] to detect all faces within each frame of the periodic focal stack, and then estimate the frames of best focus. Two focus measures were evaluated: the face detection confidence value and the standard deviation of pixels within the face bounding box. For both measures, a focus value can not be assigned if the detector misses a face. In addition, the detector (and therefore both focus measures) is highly sensitive to changes in pose and expression. For our experiments, we found the first measure gave the best results.

Finally, the estimated best-focus frames allow us to track the depth of a person of interest. The precision is limited by synchronization, face detection, and focus measure performance. Figure $2(\mathrm{~g})$ shows the estimated depths over time of the focal plane and the two walking people. A moving average filter was applied to smooth high frequency noise in the depth estimates.

Refocusable videos. By sub-sampling a periodic focal stack, one can refocus a video after the video is captured. Figure 2 shows frames from two videos with the focus following each person in the captured scene. Refocused videos were generated by selecting the frames captured at the times when the focal plane coincides with the depth of the person of interest. Some flickering in the refocused videos resulted due to tracking algorithm errors and a lack of synchronization between the deformable lens and the sensor.

Despite our relatively simple tracking algorithm, our results [20] demonstrate the potential of periodic focal stacks. We believe that periodic focal stacks could have potential applications in surveillance, security, and entertainment. The development of an imaging system with precise synchronization and more sophisticated tracking algorithms are interesting directions for future work.

\subsection{Space-Time Refocusing}

In Section 5.2, we showed how periodic focal stacks can be used to create refocused videos. Here we explore the information given in a single focal stack. The fact that a focal stack is not captured instantaneously can be taken advantage of to create a unique user experience for exploring a short duration of time [26]. Figure 8 shows one of several spacetime refocusing examples captured using the focal sweep system described in Section 4.2. In this example, the scene depth ranges from $200 \mathrm{~m}$ to $280 \mathrm{~m}$ and 50 frames are captured with a frame rate of $120 \mathrm{fps}$. After capturing the focal stack, we correct for changes in magnification and perform digital frame stabilization using SIFT [16]. The DOF of the telephoto lens is very narrow, but everything in the scene comes into focus in at least one frame of the focal stack (see Figures 8(a-c)). Figure 8 (d) shows the index map that is used to facilitate refocusing. The procedure for estimating the index map was the same as proposed in [26]. As in [26], we provide an interface that allows users to interactively refocus and explore the structure and dynamics of a scene over a short time duration. Space-time refocusing results can be viewed at [20].

\section{Conclusion}

In this paper, we have shown how deformable optics can open the door for focal sweep videography. The fast response time of deformable optics enables periodic focal sweep at high frequencies. As a result, deformable lenses are particularly well suited for focal sweep cameras with large focal lengths, which would require large sensor motion using the swept-sensor approach. We described a prototype camera that uses off-the-shelf components: we attached a deformable lens to the mounting flange of a commercial lens. An interesting direction for future work is to design the entire imaging system from scratch.

We have shown three applications of focal sweep using deformable optics: 1) EDOF video for distant scenes, 2) telephoto space-time refocusing, and 3) periodic focal stack 


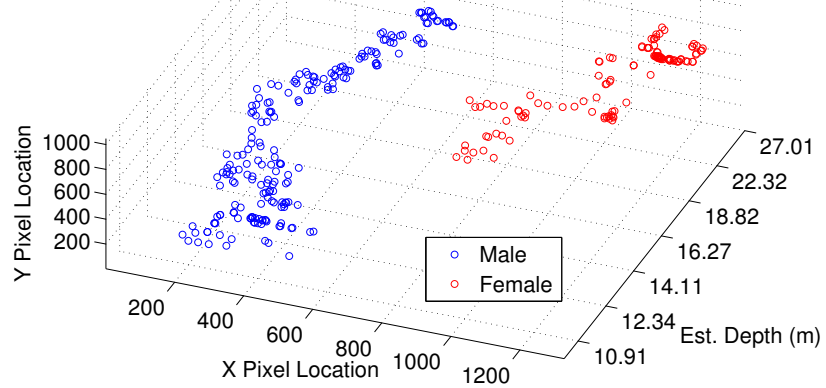

Figure 9. 3D object trajectory estimation. The estimated 3D trajectories of the two people from the periodic focal stack shown in Figure 2.

capture. We have demonstrated that periodic focal stacks can be used to create refocusable videos where focus follows a person or object of interest. In addition, information embedded in a periodic focal stack can potentially be exploited for other applications such as estimating the 3D trajectories of moving objects within the scene. Figure 9 shows the results of using a simple depth from focus algorithm (described in Section 5.2) to track the two people in Figure 2. We expect that performance can be further improved by capturing at higher frame rate (to reduce changes in pose and expression between frames), and developing more sophisticated tracking algorithms.

\section{Acknowledgments}

This research was supported in parts by ONR MURI Award No. N00014-08- 1-0638 and ONR Award No. N00014-11-1-0285.

\section{References}

[1] A. Agarwala, M. Dontcheva, M. Agrawala, S. Drucker, A. Colburn, B. Curless, D. Salesin, and M. Cohen. Interactive digital photomontage. In ACM Trans. Gr., volume 23, pages 294-302. ACM, 2004.

[2] J. Baek. Transfer efficiency and depth invariance in computational cameras. In ICCP, pages 1-8, 2010.

[3] M. Blum, M. Büeler, C. Grätzel, and M. Aschwanden. Compact optical design solutions using focus tunable lenses. In Proc. SPIE, volume 8167, 2011.

[4] O. Cossairt and S. Nayar. Spectral Focal Sweep: Extended depth of field from chromatic aberrations. pages 1-8, 2010.

[5] O. Cossairt, C. Zhou, and S. Nayar. Diffusion coded photography for extended depth of field. In SIGGRAPH, pages 1-10. ACM, 2010.

[6] E. Dowski and W. Cathey. Extended depth of field through wave-front coding. Applied Optics, 34(11):1859-1866, 1995.

[7] S. Hasinoff and K. Kutulakos. Light-efficient photography. 1(1):1, 2009.
[8] G. Hausler. A method to increase the depth of focus by two step image processing. Optics Communications, 6(1):38-42, 1972.

[9] E. Hecht. Optics. Addison-Wesley, 4th edition, 1998.

[10] A. Isaksen, L. McMillan, and S. J. Gortler. Dynamically reparameterized light fields. In SIGGRAPH, pages 297-306. ACM, 2000.

[11] S. Kuthirummal, H. Nagahara, C. Zhou, and S. Nayar. Flexible depth of field photography. PAMI, 33(1):58-71, 2011.

[12] M. F. Land and D. E. Nillson. Animal Eyes. Oxford Press, 2nd edition, 2012.

[13] A. Levin, R. Fergus, F. Durand, and W. Freeman. Image and depth from a conventional camera with a coded aperture. ACM Trans. Gr., 26(3):70-es, 2007.

[14] A. Levin, S. Hasinoff, P. Green, F. Durand, and W. Freeman. 4D frequency analysis of computational cameras for depth of field extension. In SIGGRAPH, pages 1-14. ACM, 2009.

[15] S. Liu and H. Hua. Extended depth-of-field microscopic imaging with a variable focus microscope objective. Optics Express, 19(1):353-362, 2011.

[16] D. Lowe. Distinctive image features from scale-invariant keypoints. IJCV, 60(2):91-110, 2004.

[17] R. Ng. Digital light field photography. PhD thesis, Stanford University, 2006.

[18] Omron Corporation. http://www. omron.com/, 2012.

[19] Optotune AG. http: //www . optotune.com/, 2012.

[20] Project website. http://www.cs.columbia.edu/ CAVE/projects/deformable_focal_sweep/.

[21] H. Ren, Y. Fan, S. Gauza, and S. Wu. Tunable-focus flat liquid crystal spherical lens. Applied physics letters, 84(23):4789-4791, 2004.

[22] N. Shroff, A. Veeraraghavan, Y. Taguchi, O. Tuzel, A. Agrawal, and R. Chellappa. Variable focus video: Reconstructing depth and video for dynamic scenes. In ICCP, pages 1-9. IEEE, 2012.

[23] Varioptic. http://www.varioptic.com/, 2012.

[24] Y. Zhao and Y. Qu. Extended depth of field for visual measurement systems with depth-invariant magnification. In Photonics Asia, pages 856300-85630O. International Society for Optics and Photonics, 2012.

[25] C. Zhou, S. Lin, and S. Nayar. Coded aperture pairs for depth from defocus. In $I C C V, 2009$.

[26] C. Zhou, D. Miau, and S. Nayar. Focal sweep camera for space-time refocusing. Columbia University Computer Science Technical Report, CUCS-021-12, 2012. 